\title{
Spark-SGreedy: Um Algoritmo de Escalonamento de Workflows Intensivos em Dados no Framework Apache Spark
}

\author{
Victor F. de Sousa ${ }^{1}$, Cristina Boeres ${ }^{1}$, Daniel de Oliveira $^{1}$ \\ ${ }^{1}$ Instituto de Computação - Universidade Federal Fluminense (IC/UFF) \\ \{boeres, danielcmo, victorsousa\}@ic.uff.br
}

\begin{abstract}
Resumo. Nos últimos anos, o Apache Spark vem sendo utilizado como framework para execução de experimentos científicos modelados como workflows. Por mais que represente um avanço, o Spark não foi projetado para gerenciar execuções de aplicações científicas, e seu escalonamento não considera estimativas de consumo de recursos pelas atividades do workflow. Esse artigo apresenta o Spark-SGreedy, uma proposta de algoritmo de escalonamento de workflows no Spark que usa dados de proveniência (histórico) para analisar a previsão de consumo de recursos das atividades do workflow e escaloná-las de acordo com tal previsão.
\end{abstract}

\section{Introdução}

O uso de simulações computacionais de larga escala para executar experimentos científicos ganhou importância nos últimos anos [de Oliveira et al. 2019]. Tais experimentos são compostos pelo encadeamento de programas que realizam operações sobre os dados cientıficos a serem analisados, tais como carga (data loading) e processamento (data processing). Uma abstração utilizada para modelar esses experimentos são os workflows. Um workflow é representado por meio de um DAG, onde os vértices representam as atividades (programas) que executam operações sobre os dados, enquanto as arestas representam as dependências entre elas [de Oliveira et al. 2019]. Muitos workflows são de larga escala, e podem executar por horas ou dias, dependendo do volume de dados de entrada. Dessa forma, requerem execuções em ambientes de Processamento de Alto Desempenho (PAD) aliados à aplicação de técnicas de paralelismo. Eles são comumente executados em Sistemas de Gerência de workflows (SGWfCs). Entretanto, os SGWfs possuem uma curva de aprendizado alta e nem todo workflow pode ser modelado nos SGWfs de forma simples.

Uma opção interessante aos SGWfs para a execução de workflows é o uso de Ambientes de Computação Escalável e Intensiva em Dados (i.e., DISC) e seus frameworks. Diferentemente dos ambientes PAD (que são centrados em computação), os ambientes DISC [Bryant 2011], são centrados em dados e concentram-se na tolerância à falhas e escalabilidade usando clusters de máquinas com hardware comum (i.e., commodity). Os ambientes DISC se baseiam em operadores de processamento de dados (Map, Reduce, etc). Muitos frameworks DISC se encontram disponíveis para uso, como o Apache Spark [Zaharia et al. 2016], relevantes atualmente uma vez que permite o processamento de dados em memória. Apesar de frameworks DISC representarem um enorme avanço no que tange o apoio a experimentação cientıfica, ainda existem diversas barreiras para seu uso. Os frameworks DISC não foram originalmente projetados para gerenciar workflows, que possuem requisitos diferentes de aplicações comerciais no que tange a execução, e principalmente o escalonamento de atividades. O escalonamento padrão do Spark não considera estimativas de consumo de memória e CPU das atividades. Além disso, algoritmos tradicionais de escalonamento como o HEFT [Topcuoglu et al. 2002] e o SGreedy [de Oliveira et al. 2012] não podem ser portados diretamente para o Spark, pois não consideram dados em memória nem as características de ambientes DISC. 
Assim, o objetivo do trabalho apresentado nesse artigo é desenvolver um algoritmo de escalonamento de workflows no framework Spark chamado Spark-SGreedy, de modo a apoiar transversalmente as diferentes disciplinas científicas que migram seu processo científico para projetos baseados no Spark. Exemplos de workflows que podem se beneficiar deste tipo de algoritmos de escalonamento são os workflows para geração de árvores filogenéticas. A Seção 2 discute sucintamente a abordagem proposta e a Seção 3 apresenta as conclusões.

\section{Abordagem Proposta: O Spark-SGreedy}

Para discutirmos um algoritmo de escalonamento de workflows no Spark, devemos primeiramente definir formalmente um workflow. Um workflow $W$ pode ser definido por meio de um DAG, onde os nós $A=\left\{a_{1}, a_{2}, \ldots, a_{n}\right\}$ representam as atividades e as arestas Dep representam a dependência de atividades entre a atividade em $A$. Dado $a_{i} \mid(1 \leq i \leq n)$, e seja $I=\left\{i_{1}, i_{2}, . ., i_{m}\right\}$ os dados de entrada para uma atividade $a_{i}$, então input $\left(a_{i}\right) \subset I$. Além disso, consideremos $O$ como o conjunto de dados de saída produzidos por uma atividade $a_{i}$, então output $\left(a_{i}\right) \subset O$. As dependências entre duas atividades $a_{i}$ e $a_{j}$ é representada por $\operatorname{dep}\left(a_{i}, a_{j}\right) \leftrightarrow \exists O_{k} \in \operatorname{input}\left(a_{j}\right) \mid O_{k} \in \operatorname{output}\left(a_{i}\right)$. De forma a escalonar atividades de workflows no Spark considerando consumo de recursos de cada atividade do workflow, devemos possuir dados históricos que nos ajudem a estimar o consumo de cada atividade para realizarmos o escalonamento.

Recentemente [Guedes et al. 2020] propuseram uma extensão do Spark chamada SAMbA. O SAMbA coleta dados históricos da execução de aplicações Spark, chamados dados de proveniência [Freire et al. 2008]. A partir da proveniência, é possível estimar o tempo de execução de atividades considerando os mecanismos em memória do Spark, como os RDDs e Data Frames. Todos os dados coletados pelo SAMbA estão disponíveis para consulta em um banco de dados. Assim, a proposta de algoritmo de escalonamento para o Spark implementada nesse artigo é uma extensão do algoritmo SGreedy [de Oliveira et al. 2012], chamada de Spark-SGreedy, que é apresentado no Algoritmo 1. O Spark-SGreedy recebe como entrada, o workflow a ser executado, um prazo limite, um orçamento limite e pesos para serem aplicados nos critérios de tempo de execução e custo financeiro. O algoritmo é guloso e sempre que uma máquina se encontra ociosa, uma nova atividade é enviada para o seu processamento. A escolha da melhor atividade para a máquina ociosa se dá por meio do cálculo do tempo de execução $T_{n}\left(a_{i}, v m_{j}\right)$ e seu custo financeiro $F_{n}\left(a_{i}, v m_{j}\right)$. É importante ressaltar que $T_{n}\left(a_{i}, v m_{j}\right)$ é calculado de acordo com a estrutura usada no Spark, seja ela RDD ou Data Frame. Todas as possíveis atividades são avaliadas e a que apresentar o valor mínimo para esse cálculo do custo é a escolhida. Esse processo se repete sempre que uma máquina ficar ociosa ou até que todas as atividades do workflow sejam executadas.

O desenvolvimento do Spark-SGReedy é um trabalho em andamento, mas experimentos preliminares com a execução do workflow Montage (http: / / montage. ipac. caltech . edu/) mostraram que a demanda por recursos e o tempo de execução de suas atividades são bastante heterogêneos, o que justifica um algoritmo de escalonamento como o Spark-SGreedy. Por exemplo, a atividade $m$ ProjectPP possui tempo de execução médio 13,99 min (desvio padrão de 2,12), enquanto que a atividade mBackground possui tempo execução médio de 10,64 min (desvio padrão 1,25), mas com um consumo de memória $125 \%$ maior que a atividade $m$ ProjectPP. Experimentos futuros utilizarão o Montage como benchmark.

\section{Conclusões}

Os frameworks DISC como o Apache Spark vêm sendo utilizados para execução de experimentos científicos modelados como workflows. Apesar de representar um avanço, os algorit- 
mos de escalonamento do Spark não consideram características importantes de atividades de workflows científicos como consumo de CPU e memória. Esse artigo apresentou a proposta do Spark-SGReedy, um algoritmo de escalonamento de atividades de workflows que usa dados de proveniência (histórico) como base de forma a considerar requisitos das atividades no processo de escalonamento. O Spark-SGreedy é um trabalho em andamento, mas avaliações parciais mostraram que a heterogeneidade de consumo de recursos de workflows justifica o seu desenvolvimento.

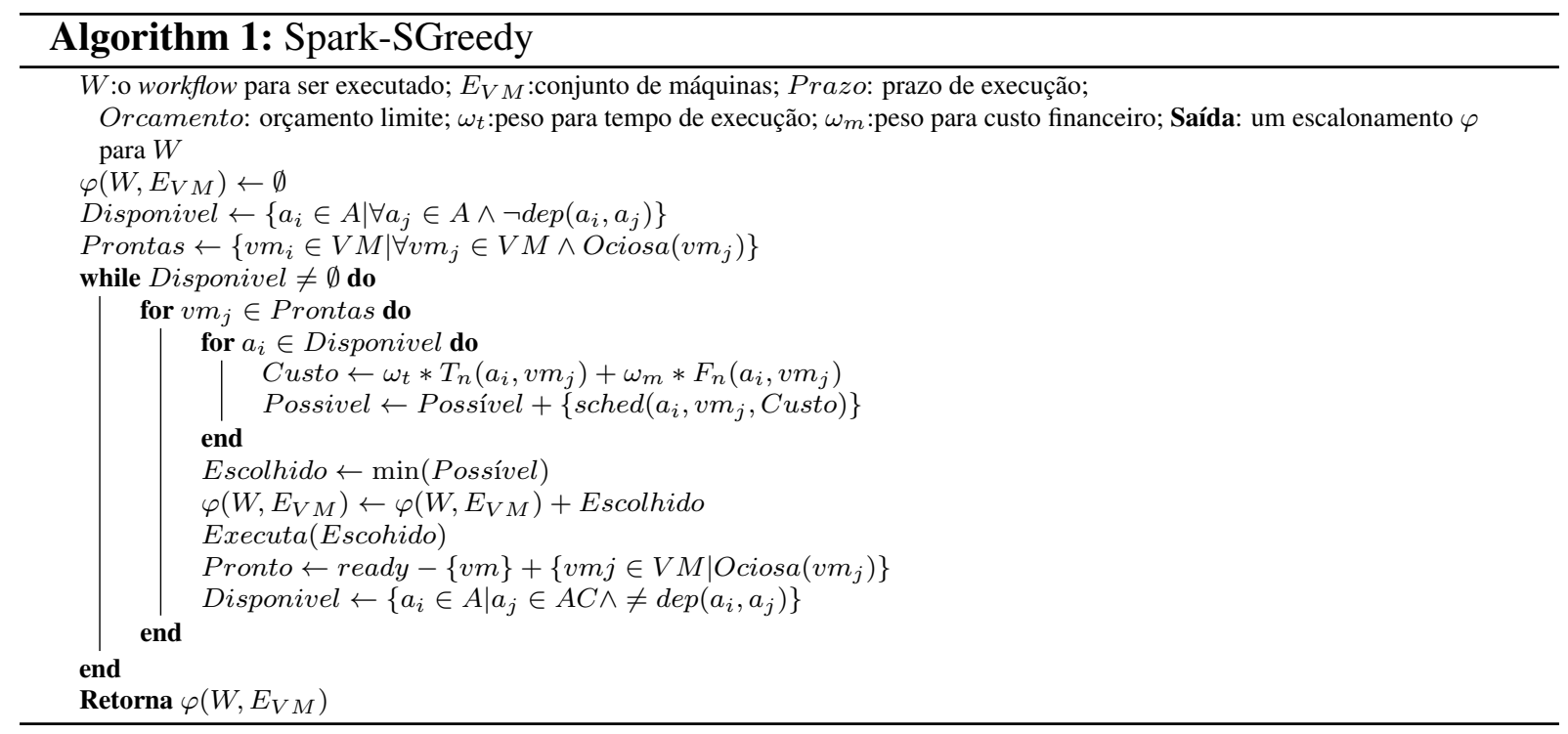

\section{Referências}

Bryant, R. E. (2011). Data-intensive scalable computing for scientific applications. Computing in Science Engineering, 13(06):25-33.

de Oliveira, D., Ocaña, K. A. C. S., Baião, F. A., and Mattoso, M. (2012). A provenance-based adaptive scheduling heuristic for parallel scientific workflows in clouds. J. Grid Comput., 10(3):521-552.

de Oliveira, D. C. M., Liu, J., and Pacitti, E. (2019). Data-Intensive Workflow Management: For Clouds and Data-Intensive and Scalable Computing Environments. Synthesis Lectures on Data Management. Morgan \& Claypool Publishers.

Freire, J., Koop, D., Santos, E., and Silva, C. T. (2008). Provenance for computational tasks: A survey. Comput. Sci. Eng., 10(3):11-21.

Guedes, T., Martins, L. B., Falci, M. L. F., Silva, V., Ocaña, K. A. C. S., Mattoso, M., Bedo, M. V. N., and de Oliveira, D. (2020). Capturing and analyzing provenance from spark-based scientific workflows with samba-rap. Future Gener. Comput. Syst., 112:658-669.

Topcuoglu, H., Hariri, S., and Wu, M. (2002). Performance-effective and low-complexity task scheduling for heterogeneous computing. IEEE Trans. Parallel Distributed Syst., 13(3):260274.

Zaharia, M., Xin, R. S., Wendell, P., Das, T., Armbrust, M., Dave, A., Meng, X., Rosen, J., Venkataraman, S., Franklin, M. J., Ghodsi, A., Gonzalez, J., Shenker, S., and Stoica, I. (2016). Apache spark: a unified engine for big data processing. Commun. ACM, 59(11):5665. 